\title{
Chapter
}

\section{Reporting medication errors and near misses}

\author{
Sheena Williamson
}

\section{Medication safety incidents}

\section{Introduction}

Between January 2005 and June 2006 there were 59802 medication safety incidents reported via the National Reporting and Learning System (NRLS) in England and Wales. Medication incidents are the second most commonly reported incident next to patient accidents (NPSA, 2007).

Although there has been an increase in reporting over the last 3 years, literature suggests gross inconsistencies and substantial under-reporting from a large number of NHS organizations (NPSA, 2007). This has been borne out in a systematic review of international literature from 12 countries suggesting the average rate of underreporting of adverse drug events as high as $94 \%$ (Hazell \& Shakir, 2006).

A significant proportion of low reporting or non-reporting has arisen from primary care organizations with only $4.9 \%$ of the total medication incidents reported to the NRLS coming from the primary care setting.

The aim of this chapter is to define what is meant by medication safety incidents and to examine where errors are likely to occur within the medication process, including a brief overview of some of the findings in the data that are pertinent to reporting medication incidents from the National Patient Safety Agency Report (2007) Safety in doses: medication safety incidents in the NHS. The main section in the chapter consists of guidance on how to report medication incidents, utilizing the recommendations from NPSA on how to improve reporting.

\section{Terms and definitions}

The National Patient Safety Agency (NPSA) has defined a patient safety incident as 'any unintended or unexpected incident which could have or did lead to harm for one or more patients' (NPSA, 2004). A wide variety of terms are used in the definition and classification of medication safety incidents and it is important to understand the differences between each of these. The model in Fig. 10. 1 demonstrates the correlation between the terms explained below.

Medication errors are broadly defined as incidents in which an error has occurred somewhere in the medication process, regardless of whether any harm occurred to the patient.

Potential Adverse Drug Events (near misses) may be identified as incidents which did not cause any harm at the time but may have had the potential to cause harm. Near misses are often under reported and yet they provide rich data to help improve the management of systems to reduce risks and improve patient safety. 


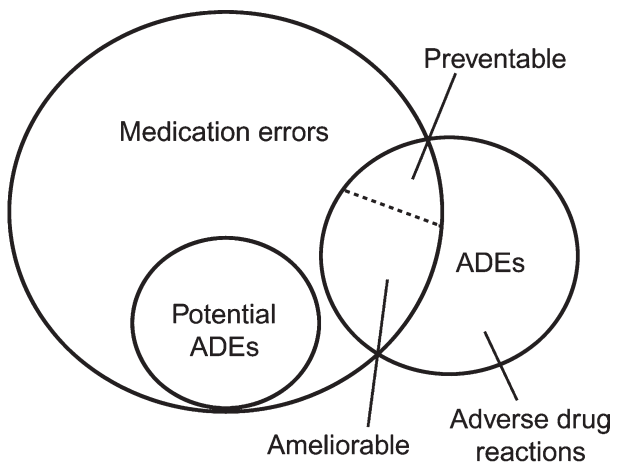

Figure 10.1. Demonstrates the correlation between medication errors, potential adverse drug events and preventable adverse drug events and non preventable adverse drug reactions (Morimoto et al., 2004)

Adverse Drug Events ADEs (actual harm from medicines) are medication incidents defined as 'any undesirable experience that has happened to the patient while taking a drug but which may or may not be related to the drug' (MHRA, 2006).

Adverse drug events may be divided into two categories.

A preventable ADE is an injury that is the result of an error at any stage throughout the medication process.

A non-preventable ADE is an injury due to a medication where there is no error in the medication process. These are also referred to as Adverse Drug Reactions (ADRs). The MHRA define ADRs as 'an unwanted or harmful reaction experienced, following the administration of a drug or a combination of medications, and is suspected to be related to the medication. The reaction may be a known side effect of the medication or it may be new and previously unrecognized'.

The NPSA collects data on all types of medication error and the MHRA collects data on adverse drug reactions via the Yellow Card System. Therefore, it is important that information is shared and data are processed between each of these organizations.

\section{Medication errors within the medication process}

The medication process is the term used to describe the process of delivering medications to patients. It consists of five stages.

Stage 1: Prescribing the medicine.

Stage 2: Dispensing the medicine.

Stage 3: Preparing the medicine for administration.

Stage 4: Administering the dose using the appropriate route and method.

Stage 5: Monitoring the effect of the medicine on the patient (NPSA, 2007).

The potential for error lies within each stage.

\section{Stage1: Prescribing errors}

Prescribing medicines is now no longer the sole responsibility of medical staff. A number of nurses, pharmacists and optometrists have undertaken education to enable them to independently prescribe almost all the medications in the British National Formulary, 
within their scope of clinical practice. Similarly, a number of allied health professions (physiotherapists, radiologists, chiropodists/podiatrists) and optometrists, nurses and pharmacists may prescribe as supplementary prescribers, in partnership with a medical practitioner.

A prescribing error may be defined as the incorrect drug selection for a patient or errors involving wrong drug, dose, quantity, indication for use or a contraindication (Williams, 2007). Prescribing errors also include illegible handwriting, misspelling of a drug with a similar name and use of abbreviations.

Prescription errors are estimated as being between $<1 \%$ and $11 \%$ of all written prescriptions (Sanders \& Esmail, 2003).

\section{Stage 2: Dispensing errors}

Dispensing is carried out in a variety of settings from hospital pharmacies, community pharmacies and some rural General Practices. One common dispensing error is selection of the wrong product, usually where there are two drugs with similar proprietary names (e.g. Losec ${ }^{\oplus}$ and Lasix ${ }^{\oplus}$ ), which may look similar when hand written. Other dispensing errors include wrong dose, wrong drug and wrong patient and some reports suggest typing errors in computerized labelling as a common cause of error in dispensing (Williams, 2007).

\section{Stage 3 and 4: Administration and preparation errors}

There is very little documented data around preparation and administration errors occurring in patients in the community. However, there is a reported wide variation in the rates of preparation and administration error within hospitals with rates varying between $3.5 \%$ and $49 \%$ (NPSA, 2007). The NPSA also suggest that this wide range reflects the differences in the definitions used to record medication error, together with methods of data collection.

Drug administration and preparation has been considered as an area of 'high risk' within nursing practice and the well-known six Rights of the Medication Use Process should be familiar to all:

- Right patient

- $\quad$ Right drug

- Right dose

- Right route

- Right time

- Right outcome

Many drug administration errors are errors of omission but also include failure to check patient identity, incorrect administration technique and administration of a wrong or expired drug (Williams, 2007).

The literature suggests that the medication error rate for administration of intravenous (IV) drugs may be as high as $25 \%$ and these errors have significant risk to patients (Bruce \& Wong, 2001).

One of the most common types of error identified is 'deliberate violation of guidelines', for example, where practitioners have injected IV medication faster than the recommended time stated in the guidelines (Williams, 2007). 


\section{Stage 5: Errors in monitoring outcome}

With an increasing elderly population, there is a subsequent rise in patients living with long-term, often complex chronic diseases. This gives rise to issues of medicines management and the need for careful monitoring of outcomes. Some patients take certain drugs, which require continuous monitoring to ensure they are taking the optimum dose for their condition at the time. Literature suggests that recommended ongoing monitoring of patients taking certain drugs is not being undertaken by healthcare professionals. The NPSA (2007) cite one study where less than one-third of patients taking diuretics were having the electrolyte levels in their blood monitored. This statement may be borne out by evidence stating diuretics as one of the medicines most commonly responsible for medicine-related admission to hospital (Pirmohamed et al., 2004; Howard et al., 2007).

The Nursing and Midwifery Council (NMC) have produced Standards for Medicines Management (2007), which sets standards for safe practice for supplying, administering and dispensing medicines to patients.

There are also Standards of proficiency for nurse and midwife prescribers (2006) and both these documents are available via the following link: http://www.nmc-uk.org/.

\section{The extent of the problem}

The Department of Health estimate that the annual cost of avoidable hospital admissions resulting from drug errors cost in the region of $£ 200-400$ million per annum.

Pirmohamed et al. (2004) carried out the largest UK study of hospital admissions data over 6 months and their findings concluded that $6.5 \%$ of all admissions were related to harm from medicines. From these findings the researchers concluded that $4.7 \%$ of all admissions were as a result of avoidable harm from medicines. The medicines most commonly responsible were aspirin, diuretics, warfarin and non-steroidal anti-inflammatory drugs. More recently, a systematic review of international literature by Howard et al. (2007) cited the drugs most commonly responsible for medicine-related admission were antiplatelets, diuretics, non-steroidal anti-inflammatory drugs and anticoagulants, which supports the UK figures.

\section{The National Patient Safety Agency and The National Reporting and Learning System}

\section{England and Wales}

The National Patient Safety Agency (NPSA) was set up in 2001 and one of its main functions was to develop the National Reporting and Learning System (NRLS), which primarily collects information on reported patient safety incidents from local risk management systems in England and Wales. The NRLS dataset is designed to collect reports from any single patient safety incident immediately after it occurs. From the data collected, themes and patterns in the types of incidents being reported may be identified including major systems failures, allowing development, promotion and implementation of solutions (WHO, 2005). 
The data are confidential and no information is held on identities of individual staff or patients and the focus is on:

- characteristics of the patient

age

sex

ethnicity

- patient outcomes

- any contributory factors

- factors which may have prevented harm.

There is also an area for free text for detailed explanations if needed.

\section{Brief summary of findings from the NRLS data}

The NPSA report Safety in Doses: medication safety incidents in the NHS (2007), was based on reports to the NRLS over a period of eighteen months, between January 2005 and June 2006. Within the time period, 59802 medication incidents were reported to the NRLS and included 'near miss' incidents. Table 10.1 shows the ten most common types of reported patient safety incident. The figures demonstrate that medication incidents are the second most frequent type of incident reported after patient accidents.

\section{Settings}

From the NPSA report (2007), it would appear that there is substantial under-reporting by NHS organizations, particularly within primary care settings. Although most prescribing and dispensing is carried out in the community, over $80 \%$ of the medication incidents reported to the NRLS came from hospital reports with only $4.9 \%$ of medication incidents being reported from the primary care setting.

Non-NHS organizations such as those run by the local authority or the voluntary sector are not connected to the NRLS at present.

Table 10.1. The ten most common types of patient safety incident (NPSA, 2007)

\begin{tabular}{lcc} 
Type of patient safety incident & Number & Percentage \\
\hline Patient accident & 278886 & 38.8 \\
Medication & 59802 & 8.3 \\
Treatment, procedure & 58921 & 8.2 \\
Access, admission, transfer, discharge (including missing patient) & 55710 & 7.8 \\
Infrastructure (including staffing, facilities, environment) & 46122 & \multicolumn{1}{c}{6.4} \\
Documentation (including records, identification) & 35533 & 4.9 \\
Documentation (including records, identification) & 34944 & 4.9 \\
Clinical assessment (including diagnosis, scans, tests, assessments) & 31644 & 4.4 \\
Consent, communication, confidentiality & 28723 & 4.0 \\
Medical device/equipment & 23389 & 3.3 \\
Total & 653674 & 91.0
\end{tabular}




\section{Age groups}

Although the patient's age was indicated in less than half of all the reports to NRLS during the 18- month period, it would appear from the data that children between the ages of 0-4 years together with older adults peaking at 75-79 years are more likely to be involved in medication incidents.

\section{Incidence occurrence during stages of the medication process}

Almost $60 \%$ of reported incidences in hospital settings occurred during administration and supply. In the community, $62.9 \%$ of incidents occurred during preparation or dispensing.

One-third of trusts (mainly primary care trusts) reported no medication incidents occurring over 6 months. More information would have been useful to support these findings as it is unclear as to whether one-third of trusts have had absolutely no medication incidents to report or whether there is a fundamental failure in their local reporting systems.

\section{Types of medication error}

Two main types of error were found in over half of the reported incidents: wrong dose omitted medicines and wrong medicines. These types accounted for almost $50 \%$ of the reported errors. Others included wrong quantity, mismatch between patient and medicine, wrong/transposed/omitted medicine label, patient allergy to treatment, wrong storage and wrong/omitted/passed expiry date.

\section{Outcome of error to patients}

Outcomes of error were reported (Piramohamed, et al., 2004) by degree of harm to patients ranging from 'no harm,' 'low, moderate or severe harm' to 'death'. From the data, it appeared that more medication incidents resulted in 'no harm' than all other incidents reported to the NRLS.

Also, there were less medication incidents resulting in 'death' than all other patient safety incidents reported. Although the proportion of reported harm is small, there is no room for complacency. Remember that these findings may also reflect under reporting.

\section{Scotland}

Following a baseline study carried out in 2005, 'A Scottish prescription - managing the use of medicines in hospitals', there were key recommendations made to NHS Quality Improvement Scotland (NHS QIS). One of these recommendations was 'to develop a national approach to collecting data on adverse incidents, including medication incidents, to allow robust trend analysis, transferable lessons and benchmarking.'

Between 2005 and 2007, QIS led an initiative to develop a standardized approach to incident and near-miss reporting across NHS Scotland.

The Scottish Government established the Scottish Patient Safety Alliance in 2007 to guide the Scottish Patient Safety Programme and promote a systematic approach 
to improving the safety of patients in hospital. It brings together the NHS, the Scottish Government, professional bodies and patient representatives in a new drive to significantly reduce adverse events and improve patient safety (Audit Scotland, 2008).

\section{Reporting patient safety incidents}

\section{What to report}

Patient safety incidents to be reported are defined by the NPSA as 'any unintended or unexpected incident which could have or did lead to harm for one or more patients receiving NHS care. The NRLS was developed by the NPSA to foster a culture of open reporting, in order to learn from adverse events.

The reports are anonymous, although it may be that the trust or Health Board may be identifiable. Names of patients and staff are removed prior to the information being stored in the database. Williams (2007) suggests there are significant increases in the reporting of medication errors where confidential 'no-fault' reporting has been implemented.

\section{When to report}

The NPSA encourages reporting of all patient safety incidents and include the following in their criteria:

- incidents you have been involved in;

- incidents you have witnessed;

- incidents which caused no harm (or minimal harm);

- incidents resulting in moderate or severe harm (including death);

- prevented patient safety incidents (near misses).

The NPSA does not have the statutory power to make incident reporting mandatory. There have been suggestions that reporting ought to be mandatory in a bid to combat under-reporting, yet the literature examining the causal factors for under-reporting does not appear to support this idea in principle. In 1976, Dr Bill Inman highlighted 'seven deadly sins', which may account for low reporting rates. These sins, as summarized by Belton et al. (1995), ranged from 'ignorance' (uncertainty of how to report), 'diffidence' (looking foolish), 'fear' (of exposure to legal liability), 'lethargy' (too busy), 'guilt' (reluctant to admit cause of harm), 'ambition' (preference to publish findings) to 'complacency' (only safe drugs are marketed).

Conversely, an attitudinal survey of Dutch physicians (Elland et al., 1999) reported the following findings:

only $26 \%$ knew which ADRs to report;

93\% thought the reaction was too well known to warrant reporting;

$75 \%$ thought the reaction was trivial;

$72 \%$ were uncertain whether the reaction was caused by a drug;

$33 \%$ did not have enough time;

$36 \%$ thought reporting was too bureaucratic;

$22 \%$ did not know how to report;

$18 \%$ were not aware of the need to report. 
From the Dutch findings, it could be argued that mandatory reporting may improve under-reporting, although it would appear that emphasis on the importance of reporting and education on pharmacovigilance is essential.

\section{Who reports?}

Any healthcare staff member may report a patient safety incident. Patients and carers may also report using a different form.

Participation in the reporting mechanism is voluntary.

\section{How to report}

Most trusts and health boards have an electronic risk management system, which has a link enabling reports to be submitted directly into the NRLS. Establishments which do not have a direct electronic link may use the electronic reporting form or 'eForm' which has been developed by the NPSA.

Individual healthcare workers may report either via their local risk management system or directly via the NPSA website.

The eForm may be accessed via the following link https://www.eforms.npsa.nhs.uk/ staffeform/.

Patients and carers may also report safety incidents via the following link: https://www.eforms.npsa.nhs.uk/eformPP/step1.do.

\section{What happens to the report?}

The information provided in each report is fed into the NPSA database. Considerable learning can be gained from the data as this provides information on 'trends and patterns in patient safety' which enables solutions to be developed.

Lessons learned are disseminated through the publication of feedback such as:

- NRLS Quarterly Data Reports;

- Patient Safety Alerts.

Incident reports are not generally made available to the public, although some trusts and health boards may provide information to the public if deemed necessary.

\section{Reporting adverse drug reactions (ADRs)}

A recent study by Pirmohamed et al. (2004), determined that ADRs were related to $6.5 \%$ of adult hospital admissions and that in $80 \%$ of those, the ADR was the direct cause of admission. The study also found that ADRs accounted for $4 \%$ of all hospital admissions resulting in a projected annual cost to the NHS of $£ 466$ million. Further findings showed that the mortality rate of patients admitted with ADRs was over $2 \%$.

Reporting of ADRs began in 1964 following the thalidomide tragedy. In 1958, Dis$\operatorname{taval}^{\circledR}$ was advertised as being an 'outstandingly safe' medication which was 'relatively free from side-effects'. It was commonly given to women during the first 3 months of pregnancy to combat nausea and sleeplessness. Following a huge rise in the number of babies being born with deformities, Distaval ${ }^{\circledR}$ was withdrawn from sale in the UK from 1961. 
As a direct result of the world-wide extent of the thalidomide tragedy, the Committee on Safety of Drugs was established in the UK in 1964 and was the world's first reporting mechanism for adverse drug reactions. In 1971, the Committee on Safety of Drugs became the Committee on Safety of Medicine (CSM) and in 2005, the committee became the Commission on Human Medicines (CHM). It is an expert committee within the Medicines and Healthcare Products Regulatory Agency (MHRA) whose duties include:

- advising Ministers on matters relating to human medicinal products;

- advising the Licensing Authority (LA), including giving advice in relation to the safety, quality and efficacy of human medicinal products;

- considering representations made in relation to the Commission's advice by an applicant or by a licence or marketing authorisation holder;

- promoting the collection and investigation of information relating to adverse reactions for human medicines (except for those products that fall within the remit of $\mathrm{ABRH}$ or $\mathrm{HMAC}$ ) for the purposes of enabling such advice to be given (MHRA, 2008).

\section{How to report adverse drug reactions}

\section{The Yellow Card scheme}

The Yellow Card scheme is a British initiative run by the MHRA and the CHM and it is used to gather information from anybody, health professionals and the general public, on suspected side effects or ADRs from:

- prescription medicines;

- herbal remedies;

- over-the-counter medicines;

- ADRs suspected to be caused by unlicensed medicines in cosmetic treatments.

The MHRA and its predecessor organizations have collected reports of suspected adverse drug reactions through the Yellow Card scheme for over 40 years. Since the establishment of the scheme, over 500000 UK reports have been collected. The Yellow Card scheme acts as an early warning system for the identification of previously unrecognised reactions and enables identification of risk factors, outcomes of the ADR and other factors that may affect clinical management.

The continued success of the scheme is dependent on the vigilance of UK healthcare professionals and their willingness to report suspect ADRs. Every report can make a difference.

The MHRA and CHM also have five Yellow Card Centres whose role focuses on follow-up of reports in their areas as this has been shown to improve follow-up rates.

- Liverpool

- Cardiff

- Edinburgh

- Newcastle-upon-Tyne

- Birmingham. 


\section{Access to Yellow Cards}

Paper copies of Yellow Cards may be found in copies of the British National Formulary (BNF), the Nurse Prescribers' Formulary (NPF), the British National Formulary for Children (BNFC), the Monthly Index of Medical Specialties (MIMS) Companion and from the Association of the British Pharmaceutical Industry (ABPI) Compendium of Data Sheets and Summaries of Product Characteristics.

Electronic Yellow Cards may be accessed via the MHRA website www.yellowcard. gov.uk.

\section{Who can report via the Yellow Card scheme?}

All healthcare professionals and members of the general public may submit reports. It should be noted that patient reporting is undertaken using a different form within the MHRA web site.

\section{What should be reported via the Yellow Card scheme?}

Any suspected ADR should be reported through the Yellow Card scheme. If there is any doubt around whether a patient has indeed suffered from an ADR, it is good practice to report this anyway. It is important to remember that the cause does not need to be established beforehand.

It is essential to report all reactions for the following:

- black triangle drugs;

- reactions in children;

- all serious reactions from established drugs and vaccines including those that are:

. fatal

- life-threatening

- disabling or incapacitating

- result in prolonged hospitalization;

- cause congenital abnormality;

- medically significant.

- Areas of particular importance including:

- delayed drug effect;

- elderly patients;

- ADRs associated with herbal remedies;

- congenital anomalies.

The MHRA produced the flowchart below to clarify when to report an ADR

\section{Black triangle drugs}

Black triangle products are new drugs and vaccines, which are being monitored more intensively in order to confirm the risk/benefit profile of the product. They are indicated in reference texts by the inverted black triangle symbol (insert black triangle symbol here).

Healthcare professionals are encouraged to report all suspected ADRs for black triangle drugs, regardless of the seriousness of the suspected reaction. Black triangle products are usually new drugs but black triangle status can also be applied to: 
- a new combination of active substances (even though those substances have been previously licensed);

- administration via a new route of administration or drug delivery system/device;

- A significant new indication, which may alter the established risk/benefit profile of the product.

There is no standard time for a product to retain black triangle status. However, an assessment is usually made following 2 years of post-marketing experience and the black triangle symbol is not removed until the safety of the drug is well established (MHRA, 2008).

A list of black triangle products is available on the MHRA website: www.mhra.gov.uk.

\section{Serious reactions of particular importance}

All serious reactions should be reported via the Yellow Card scheme; however, there are some areas of particular importance to the MHRA and these include children, the elderly, delayed drug effects, congenital anomalies and ADRs associated with herbal medicines.

\section{Reactions in children}

It is important to remember that many medicines routinely used for children are not licensed for use in this age group. All suspected ADRs that occur in children under 18 years of age should be reported, regardless of whether the medicine is licensed for children or not. Monitoring of drug safety is essential for this age group as children are not generally exposed to medicines within clinical drug trials.

\section{Reactions in elderly patients}

The number of people over 65 years of age continues to grow. A high proportion of these patients receive medicines on a regular basis. The physiological changes that occur naturally with ageing have an impact on pharmacokinetics and pharmacodynamics related to drug therapy in these patients. The elderly are more susceptible to the therapeutic and adverse effects of drugs. Elderly patients often receive multiple numbers of medicines for their multiple disease states, which increases the risk of drug side effects and drug interactions. It is therefore important to monitor the safety of medicines in this age group.

The National Service Framework for Older People (2001) describes how to maximize the benefits of medicines and how to avoid excessive, inappropriate or inadequate consumption of medicines by older people.

\section{Delayed drug effects}

Delayed effects may be more difficult to monitor as they may not manifest until months or years after exposure to a particular drug. The MHRA request that any suspicion of such an association is reported.

\section{Congenital anomalies}

If a baby is born with an abnormality, or if a pregnancy results in a malformed aborted fetus, any suspicion of an adverse drug reaction during the pregnancy must be reported. 


\section{Herbal medicines}

There is a history of herbal products being perceived as 'safe' as they are thought of as being natural products. From a safety point of view, it is worth considering these products in the same way as conventional medicines for the following reasons.

- Often they have not undergone adequate clinical trial testing.

- They may not have a product licence.

- They can cause unexpected adverse reactions and interact with other medicines there is often little documented evidence with regard to adverse reactions and interactions (Barnes et al., 2003).

\section{What other information is needed?}

There are four crucial pieces of information needed when reporting an ADR:

\section{- Suspected drug/drugs}

- Name of drug

- Route of administration

- Daily dose

- Dates of administration

- Suspected reaction

- Suspected drug reaction (including diagnosis if appropriate)

- Date of reaction

- Seriousness of reaction

- Any treatment given for the reaction

- Patient details

- Sex of patient

- Age at time of reaction

- Patient's weight(if known)

- Patient's initials and local identification number

- Reporter details

- Full name and address

Any other information which may be relevant to the case should be reported.

\section{Improving reporting of medication incidents}

\section{NPSA seven priority areas for action}

The NPSA (2007) have challenged the NHS organizations in their report, 'Safety in doses: medication safety incidents in the NHS', over some of their findings highlighting weaknesses in current medication practice.

In order to improve patient safety, they have recommended seven priority actions for all staff to implement and have determined priorities for both individual healthcare professionals and for NHS organizations. 


\section{Increase reporting and learning from medication incidents}

Recommendations to individual healthcare professionals.

- Report any medication incidents either directly to NRLS via the website or through the local risk management system.

- Ensure reporting medication incidents are included as an objective in personal development plans (PDPs).

- Use reflection and analysis of incidents to enhance your objectives for your PDP.

Recommendations for NHS organizations.

- Ensure commitment at strategic level to improving patient safety.

- Increase reporting of medication safety incidents.

- Ensure quality assurance processes are in place by engaging the senior pharmacists with chief executive, medical and nursing directors to ensure incident reports are completed and reviewed.

- Form a multidisciplinary group to carry out reviews of medication incidents, audit and initiate action to minimize risk.

- Ensure regular feedback is given to all healthcare workers.

- Produce a widely available annual report that summarizes incident reports and the learning gained from them.

\section{Implement NPSA safe medication practice recommendations}

Since 2001, the NPSA has produced guidance in safe medication practice, particularly to help minimize the risks associated with the 'high-risk' medication practices. These may be found on the NPSA website under Patient Safety Alerts http://www.npsa.nhs. uk/patientsafety/alerts-and-directives/alerts/.

Recommendations for individual healthcare professionals.

- Read and implement the guidance produced by NPSA to help minimize risk in practice from these high-risk medications/medication practices.

Recommendations for NHS organizations.

- Monitor and audit the NPSA guidance and evaluate improvements following inclusion of the information.

- Share results of evaluations with Healthcare Commission and NHS litigation organizations.

\section{Improve staff skills and competencies}

The NPSA has developed multidisciplinary work-based competences as part of recent patient safety alerts such as anticoagulant therapy, the use of injectable medicines and paediatric infusions. These e-learning competences are designed to help healthcare professionals to acquire essential knowledge to allow them to practise safely.

Recommendations for individual healthcare professionals and NHS organizations.

- Use the e-learning packages to help develop competence in the safe use of medicines.

- Identify other competences which may be improved in the future by education and training. 


\section{Minimize dosing errors}

From the NPSA report (2007) it would appear that dosing errors are the most frequently reported medication incident, with older adult and children being more commonly involved.

Recommendations for individual healthcare professionals.

- Make full use of all accessible, essential information (national and local medicines information services and therapeutic protocols) when prescribing, dispensing, preparing, administrating or monitoring medicines.

- Always undertake the required safety checks on dosages.

- If drug calculations are necessary, always have another member of staff calculate the dose independently from you.

- Ensure all clinical monitoring and dosage adjustments are in place as and when required.

- During prescribing, dispensing or administering medicines, ensure awareness of previous doses and any changes to patient's condition that may result in an alteration to the dose of medicine.

Recommendations for NHS organizations.

- Dosing errors must be audited and analysed to identify common local risks.

- Ensure all appropriate staff have access to essential information to enable them to prescribe, dispense, prepare, administer and monitor safely.

- Review local and national policies to ensure they provide accurate guidance to minimize risk.

- Ensure staff have access to (where available) calculation charts, software, syringe drivers and ready-to-use products to avoid complex dose calculations.

\section{Ensure medicines are not omitted}

The second largest cause of reported medication incidents, according to the NPSA report (2007) is omitted medicines. The report highlighted serious and fatal outcomes for omissions of certain drugs such as anticonvulsants and insulin.

Recommendations for healthcare professionals.

- Do not ignore omissions caused by prescribing, dispensing or administering errors and report all serious omissions as a medication incident.

Recommendations for NHS organizations.

Evaluate and audit all reports of omissions and delays. Results should be used to target areas of high reporting and to ensure systems are appropriate to reduce likelihood for the future.

- Review medicine storage and supply chains regularly.

\section{Ensure the correct medicines are given to the correct patient}

This section deals with two 'human error' categories:

mis-selection of a drug

mis-identification of a patient. 
The NPSA is working with the pharmaceutical industry to improve medication package design and labelling to avoid confusion and mis-selection of drugs, which may occur at any time in the medication process.

Recommendations for healthcare professionals.

- Ensure awareness of drug names that look and sound alike. Colleagues should be alerted upon discovery of a drug that has a similar name to another.

- Store medicines that look or sound alike in different locations.

- Use labels that alert other users to the risk.

- Double check with a colleague before administration.

- Liaise with pharmacy department to use medicines with safer designs to minimize risk.

- For patients in hospital, avoid mis-identification of these patients by checking identification - using auto-ID technology (if available), hospital number, NHS number, date of birth and address where necessary.

Recommendations for NHS organizations.

- Be aware of the risks of medications that look and sound alike. Improve the medication system.

- Review reports of wrong medicine and wrong patient selection and identify medications most frequently mis-selected. Findings should be used to improve systems and minimize risk to patients.

- Improve and develop purchasing for safety policies and medicines.

- Ensure policies are in place for separate storage, alert labels and double checking to help minimize risk.

- Consider auto-ID technology.

\section{Document patient's medicine allergy status}

As a significant number of reported serious incidents involved patients who had a known allergy to medication, the NPSA have made the following recommendations.

Recommendations for healthcare professionals.

- Ensure medication allergy status is documented in patient's notes.

- Do not prescribe, dispense or administer a drug if you are unsure of the patient's allergy status.

Recommendations for NHS organizations.

- Audit the frequency of medication incidents involving patient allergy.

- Ensure all electronic prescribing and dispensing systems utilize a record of the patient's allergy status so that an alert is given prior to prescription or dispensing.

- For patients in hospital, use coloured wristbands for alerting staff to patients with known medication allergy.

- Ensure that systems are developed locally to ensure staff understand that some combinations of drugs may contain penicillin and could cause serious harm to patients with penicillin allergy (e.g. co-amoxiclav, co-fluampicil). 
The NPSA also recommend that healthcare commissioners ensure that reporting and learning of medication incidents is a requirement for all commissioned services who are involved in the use of medicines.

All commissioned NHS organizations are also required to submit an Annual Report on their medication practice.

\section{NPSA and NHS confederation in March 2008}

\section{Five key changes to improve patient safety by strengthening reporting and learning}

In March 2008, the NPSA, together with an NHS confederation, consisting of 20 of the organizations with the highest reporting figures, shared their experiences and produced a briefing outlining five organizational changes to improve patient safety reporting.

The organization changes are briefly summarized below:

Change 1: Give feedback to staff.

It was felt that feedback on reporting was encouraging for staff and this motivated them to continue to report.

Methods of feedback included newsletters and case study reports among others.

Change 2: Focus on learning.

The focus of the 'blame culture' needs to shift so that the focus is more about what has been learned from incidents so that improvements may be made in that particular area to improve patient safety.

Change 3: Engage frontline staff.

There are suggestions about employing clinicians as 'safety champions' to flag up the issue at grass roots levels.

Change 4: Make it easy to report.

Access to forms needs to be easy with one well-designed form for all incidents. Organizations using web-based forms found improved consistency and efficiency in their reporting patterns.

Change 5: Make reporting matter.

It seemed that the organizations who robustly reported incidents had clear lines of strong leadership and their data were used to influence their decisions at a high level.

\section{Summary}

In conclusion, it has been well documented that medication errors are seriously underreported and that the total number of reports have largely remained unchanged since the late 1980s. All healthcare professionals have a responsibility to report medication errors to help to reduce further recurrence. In order to improve reporting rates, there needs to be an increase in awareness of the need to report, together with standardized consistent education on the importance of pharmacovigilance as part of continuing professional development for all healthcare workers.

It is gratifying to see that, in recent years, the proportion of reports being submitted by nurses and pharmacists has increased (MHRA, 2008). With increased participation by other healthcare professionals in medications management and prescribing, perhaps it is now time for mandatory reporting of medication errors. 


\section{References}

Audit Scotland (2008) A Scottish Prescription: managing the use of medicines in hospitals follow-up study. Project Brief (June 2008). http://www. audit-scotland.gov.uk/docs/fwd/pb_ managing_medicines_followup.pdf

Barnes J, Anderson LA, Phillipson JD. (2003). Herbal Therapeutics. (10) Herbal Interactions. Pharmaceutical Journal, 270(7233), 118-21.

Belton K, Lewis S, Payne S, Ralins MD, Wood SM. (1995). Attitudinal survey of adverse drug reaction reporting by medical practitioners in the United Kingdom. British Journal of Clinical Pharmacology, 39, 223-6.

Bruce J, Wong I. (2001) Parenteral drug administration errors by nursing staff on an acute medical admissions ward during day duty. Drug Safety, 24(11), 855-62.

Department of Health (2001) National Service Framework for Older People. London, Department of Health.

Elland I, Belton K, van Grootheest A, Meiners AP, Rawlins MD, Stricker BHCh. (1999). Attitudinal survey of voluntary reporting of adverse drug reactions. British Journal of Clinical Pharmacology, 48(4), 623-7.

Ferner RE, Aronson JK. (2000). Medication errors, worse than a crime. The Lancet, 355(9208), 947-8.

Hazell 1, Shakir SA. (2006). Under reporting of adverse drug reactions: a systematic review. Drug Safety, 29, 385-96.

Howard RL, Avery AJ, Slavenburg S. et al. Pirmohamed M (2007). Which drugs cause preventable admissions to hospital?
A systematic review. British Journal of Clinical Pharmacology, 63(2), 136-47.

MHRA website, Safety information: What to report, Causality. At www.mhra.gov.uk/ home/idcplg?IdcService=SS_GET_ PAGE\&;nodeId=754(accessed July 2008).

Morimoto T, Gandhi T, Seger A, Hsieh T, Bates D. (2004). Adverse drug events and medication errors: detection and classification methods. Quality and Safety in Health Care, 13, 306-14.

National Patient Safety Agency (2004). Seven Steps to Patient Safety. London: NPSA.

National Patient Safety Agency (2007) Safety in Doses: medication safety incidents in the NHS. London: NPSA.

National Patient Safety Agency Briefing (2008). Act on reporting: five actions to improve patient safety reporting. Issue 161.

Pirmohamed M, James S, Meakin S. et al. (2004). Adverse drug reactions as cause of admission to hospital: prospective analysis of 18,820 patients. British Medical Journal, 329, 15-19.

Sanders J and Esmail A. (2003). The frequency and nature of medical error in primary care: understanding the diversity across studies. Family Practice, 20, 231-6.

World Health Organization (2005). WHO draft guidelines for adverse event reporting and learning systems. Geneva: WHO. http://www.who.int/patientsafety/ events/05/Reporting_Guidelines.pdf

Williams DJP (2007) Medication errors. Journal of the Royal College of Physicians Edinburgh, 37, 343-6. 
- 